\title{
Emission Control in Diesel Engine Using Magnesium as a Catalyst
}

\author{
S.Yuvaraja, S.Venkatesh, P.karthikeyan, R.kumarasubramanian
}

\begin{abstract}
Most cities in the world are subjected to rapid urbanization and a majority of the country's population is estimated to move towards the cities within a span of the two decades. Majority of Population in all over country expects to live in a city, Such that most cities face rapid Urbanization. The fast growth in urban cities has stemmed in an incredible growth in the quantity of motor vehicles. In some major cities, the motor vehicles are doubled in the last decade. The numbers of vehicles used in cities have almost tripled its usage in a number of Automobiles by few decades. The majority of environmental pollution is caused by two-wheelers in large number. This results in a massive environmental pollution. A serious prerequisite for most effective precautionary measures has to be carried out. The emission controlled by dual techniques to control the pollution specifically, pre-pollution and post pollution control. Here the investigation is conceded out by establishing the post pollution control method for the two-wheeler automobiles via magnesium as a catalyst. To accomplish this objective, a pioneering proposal of catalytic converter is offered using magnesium as a catalyst for two-wheeler automobiles. The projected technique is precise operational in the deterrence of conservational pollution contributed from two-wheeler automobiles. It encompasses the consumption of magnesium which is economical than the, palladium, platinum and rhodium nano-particles used in automobiles.
\end{abstract}

\section{INTRODUCTION}

In this Investigation, numerous hydroxides and metal oxides with their complex oxides containing $\mathrm{Li}$ were explored. It was carried out for their $\mathrm{CO} 2$ absorption properties at different temperatures to obtain a better understanding of the related mechanism.

The inflection points in the TG curves, it was found that the major reaction changes from $\mathrm{CO} 2$ absorption to $\mathrm{CO} 2$ release [1]. The Recent research were carried out on magnesium/magnesium alloys based corrosion that occurs in the industry. Except for reviewing the kinds of corrosion on galvanic corrosion, stress corrosion, and corrosion fatigue, its corresponding mechanisms are also explained, such as the influence of metallurgical factors, and Environmental factors. Furthermore, the protection of magnesium/magnesium alloys corrosion is presented, for instance, surface treatment/modification is coating and soon. Also, because the

Revised Manuscript Received on July 22, 2019

S.Yuvaraja, Assistant Professor, School of Mechanical Engineering, Sathyabama Institute of Science \& Technology, Chennai, India.

S.Venkatesh, Assistant Professor, School of Mechanical Engineering, Sathyabama Institute of Science \& Technology, Chennai, India.

P.Karthikeyan, Assistant Professor, School of Mechanical Engineering, Sathyabama Institute of Science \& Technology, Chennai, India.

R.Kumarasubramanian, Assistant Professor, School of Mechanical Engineering, Sathyabama Institute of Science \& Technology, Chennai, India. continuing challenges associated with the use of magnesium alloys, more considerations about future applications and research directions are given according to the properties improvement of magnesium alloys for the eco-friendly requirements [2].

In this paper, $\mathrm{Mg}$ nanoparticles were prepared in tetra-hydrofuran via lithium reduction of the corresponding Mg salt. X-ray diffraction and scanning electron microscope investigations confirm the formation of hexagonal phase $\mathrm{Mg}$ particles with an average size of $300 \mathrm{~nm}$. X-ray photoelectron spectrometer analysis indicates that the as-prepared $\mathrm{Mg}$ nanoparticles are covered with a protecting layer consisting of residue solvents, naphthalene and $\mathrm{Mg}(\mathrm{OH}) 2$, which slows down further oxidation under ambient conditions. Thermal analysis shows that the rapid oxidation and nitridation processes of the particles take place at around $500{ }^{\circ} \mathrm{C}$ and 553 ${ }^{\circ} \mathrm{C}$, respectively [3]. Impending strategies make public the take into consideration of Alternative fuels and transmission of the fuels will further help citizens to reduce vehicular emissions strategies like the use of alternative fuels and spreading awareness amongst citizens will further help reduce vehicular emissions [4].The emissions of carbon monoxide (CO), hydrocarbons ( $\mathrm{HC}$ ) and nitrogen oxides (NOx) in the atmosphere can be significantly reduced by advanced emission treatments [3].The three-way catalyst can significantly control emission from the gasoline vehicles. The ceramic and metallic substrates are the most commonly available products in the market [4]. For required high flow rates and low-pressure drop application, the monolith is the one among the most commonly used. Due to the low manufacturing cost, the monolith is the most commonly used substrate material when compared with ceramic-based materials. [5, 6]... In recent times, the metallic foil monolithic substrates seem to a more conventional one. Both substrates have a relatively low porosity that makes them inappropriate as a catalyst support. As a catalyst, both the substrates are inappropriate due to their relatively low porosity. In order to overcome this problem, the channel walls are applied with a thin porous layer of material. Which could be helpful to apply these substrates as catalyst and layer are alluded to as wash coat. A regularly utilized wash coat material is cAl2O 3 with a surface zone of around $100 \mathrm{~m} 2 / \mathrm{g}[7,8]$. Car impetuses are commonly accessible as stone monument clay as cordierite and zeolites or metal substrate. The impetus substrates beneficial utilized is made out of magnesium cordierite (2MgO.2A12O3.5SiO2) with a honeycomb structure, which gives a high geometric surface territory, covered with-alumina wash coat. This wash-coat is intended to expand the particular surface

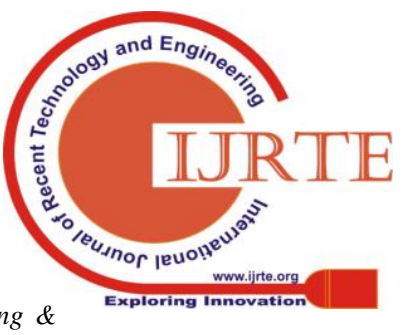




\section{Emission Control In Diesel Engine Using Magnesium As A Catalyst}

region and is the help for valuable metals, mostly palladium $(\mathrm{Pd})$, platinum $(\mathrm{Pt})$ and rhodium $(\mathrm{Rh})$, which improve the reactant oxidation and decrease of fumes poison gases from IC motor to increasingly innocuous ones, similar to water, carbon dioxide and nitrogen. Zirconium and cerium oxides are utilized in the covering by their oxygen stockpiling ability to improve reactant effectiveness [9]. Aluminium is a broadly utilized vigorous added substance to fiery materials and an added substance to fuel for air-breathing impetus [10]. In this examination, the customary exhaust system changed by covered with $\mathrm{A} 12 \mathrm{o} 3$ nanoparticles have been created to oxidize/decrease outflows from the DI diesel motor and improve the execution of the exhaust system. The benefits of this exhaust system are expressed as ease and higher substrate region which is productive to oxidize/decrease outflow when contrasted with the customary exhaust system..

\section{METHODS AND MATERIALS}

The Elemental magnesium is a rather strong, silvery-white, light-weight metal It tarnishes to some extent in a wink unembellished to expose, in spite of that, different the alkali metals, an oxygen-free tone is non-essential for storage as a remedy for magnesium is charmed by a lessen covering of oxide that is fairly impermeable and difficult to remove. Magnesium reacts round foremost at territory temperature, in all events it reacts much more slowly than calcium. As soon as lowered in power supply, hydrogen soap up respecting unnoticeably climb to presence on the come up of the metal - yet, if powdered, it reacts much more rapidly. The boomerang occurs faster more higher temperatures. Magnesium's capacity to work upon foremost depths be harnessed to supply energy and run a magnesium-based engine. Magnesium aside from reacts exothermically relating to wealthiest acids, such as hydrochloric acid $(\mathrm{HCl})$. As everywhere aluminium, zinc, and diverse succeed metals, the repercussion Prevalent $\mathrm{HCl}$ produces the chloride of the metal and releases hydrogen gas. Anent these inheritance of Magnesium, it is second-hand as a gubbins in the beneficial converter and its rebound with exhaust gases is analysed.

\section{COATING PROCESS}

Sodium silicate arrangement was utilized in wash coat material to build the covering quality. Ninety grams of sodium silicate arrangement were included into $10.0 \mathrm{gm}$ of Al2o3 nanoparticles to get $10 \%$ Al2o3 nanoparticles slurry. The slurry has been blended by utilizing a mechanical homogenizer for two hours. Covering of impetus (A12o3 nanoparticles slurry) is finished by plunge covering technique, and it has been kept in a stove at $120^{\circ} \mathrm{C}$ for 5 hours. Aluminium oxide nanoparticles served double capacities: a decrease impetus and an aluminium substance for the wash coat. Rutile type of $\mathrm{Al} 2 \mathrm{o} 3$ was picked in light of its warm dependability from $600^{\circ} \mathrm{C}$ and high strength. This property is appropriate for impetus insertion or impetus.
The synopsis of the planning of aluminium oxide nanoparticle impetus in the exhaust system is given in figure.

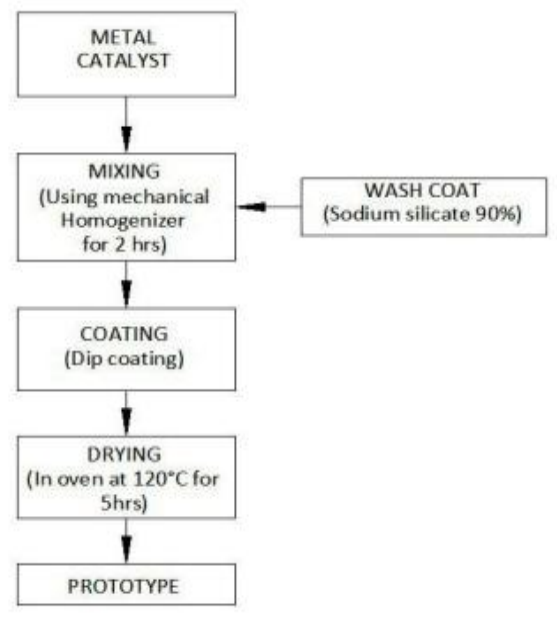

Figure2. Synopsis of the planning of aluminium oxide nanoparticle

\section{REACTION IN CATALYTIC CONVERTER}

Gases from motor fumes port went into exhaust system are alluded to as feed gases, for example, HC, CO, NOx, and $\mathrm{CO} 2$. In the exhaust system, two synthetic procedures are happening, for example, reactant decrease and synergist oxidation. In the reactant decrease process, nitrogen oxide surrenders its oxygen to shape unadulterated nitrogen. At that point, the free oxygen responds with $\mathrm{CO}$ to shape $\mathrm{CO} 2$ outflows. In the oxidation procedure, hydrocarbons and carbon monoxide keep on consuming. This happens just if there is an adequate measure of oxygen accessible for the hydrocarbons and carbon monoxide to shape with. This compound response results in the oxidation of hydrocarbons and carbon monoxide to frame carbon dioxide and water. Itemized of reactant decrease and oxidation can be found in figure.

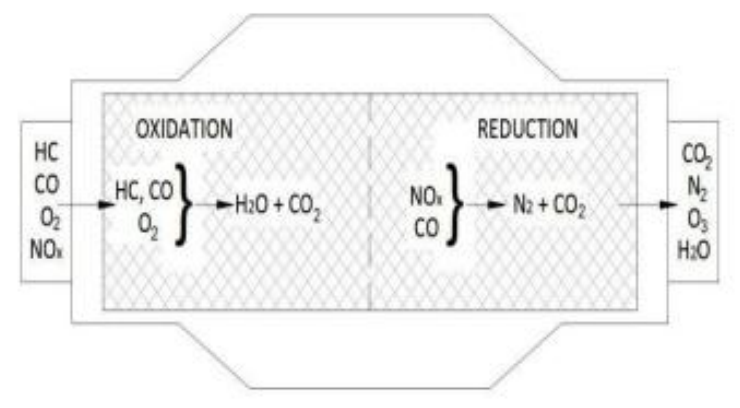

\section{FABRICATION OF CATALYTIC CONVERTER}

The design process is carried using modeling software PRO-E. The parametric modeling approach uses parameters, dimensions, features, and relationships to capture intended product behaving or and create a recipe which enables design automation and the optimization of design and product development processes. This design approach, whose product strategy is family-based or platform-driven is used by the companies, where a prescriptive design strategy is fundamental one. The resulting geometry may be complex or based upon equations based on the success of the design process 
by embedding engineering constraints and relationships to quickly optimize the design. Cero Elements and Pro provides a complete set of design, analysis and manufacturing capabilities on one or integral and scalable platform. The Solid Modeling, Surfacing, Rendering, Data Interoperability, Routed Systems Design, Simulation, Tolerance Analysis, and $\mathrm{NC}$ and Tooling Design can also be used as downstream in finite element analysis, rapid prototyping, tooling design, and CNC manufacturing. All the data's are modified between the CAD and CAM modules without conversion. The entire bill of materials (BOM) as well as the products can be modelled precisely with fully associative engineering drawings with its revision control information. The associative functionality in Cero Elements / Pro facilitates its users to make changes in the design. Product development process updates downstream deliverables automatically at any time. This concurrent engineering has the ability to Design, analysis and manufacture the engineer work in parallel and streamlines for the product development processes

\section{RESULTS AND DISCUSSION}

From the above graph, we can observe that there is a reduction in emission after implementing the catalytic converter to the motorcycle. This is tested at initial load conditions. The wire mesh's conversion efficiency was found to be $48 \%$. The wire mesh catalytic converter reduces NOx emission by $24 \%$ higher than a normal catalytic converter. This is because of the effect caused by chemical with the higher wire mesh substrate area. The catalyst intensify the reaction rate by adsorption of reactants. The activation energy for the reaction is further reduced far below its value in uncatalyzed reaction.

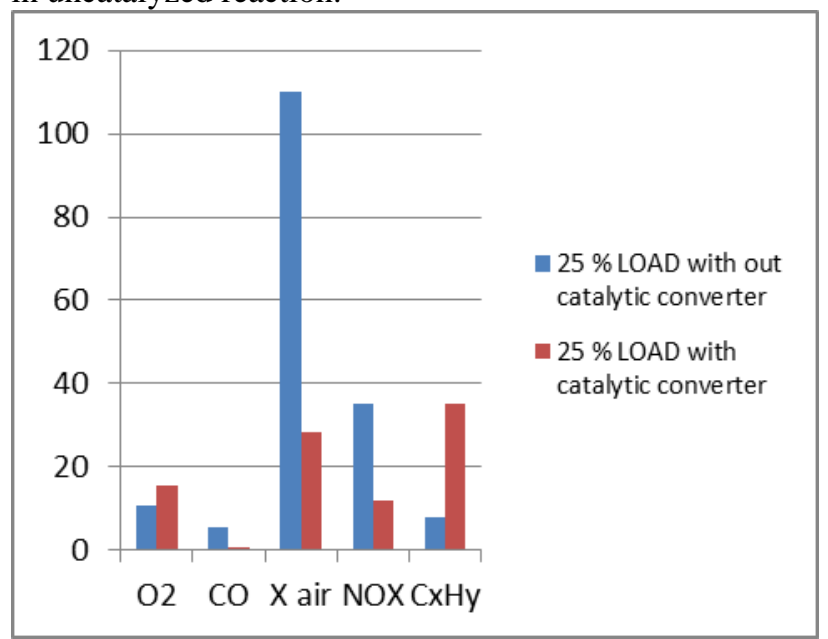

Fig 4.1 Emission Testing at Initial load 25\%
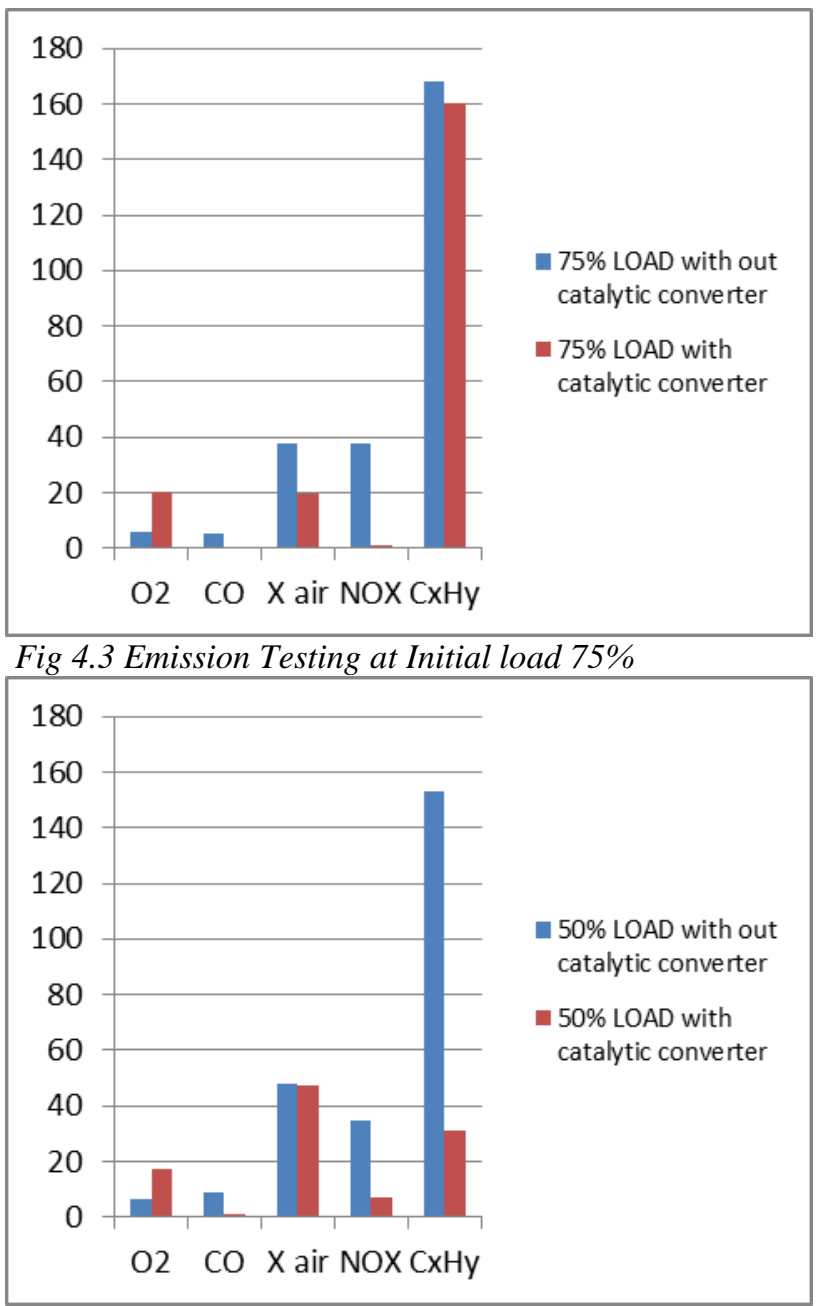

Fig 4.2 Emission Testing at Initial load 50\%

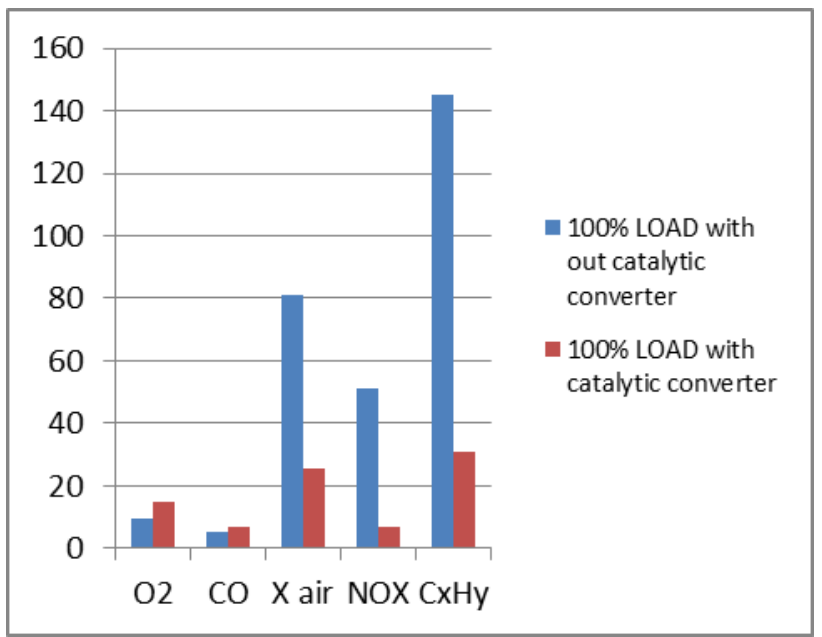

Fig 4.4 Emission Testing at Initial load 100\%

The conversion efficiency of wire mesh and OEM catalytic converter is found to be $60 \%$. The wire mesh catalytic converter reduces $41 \%$ higher $\mathrm{CO}$ emission than the original catalytic converter. The higher specific surface area of the wire mesh substrate, when it is equalled with the monolith substrate, is beneficial at higher operating temperature. The conversion efficiency of wire mesh is found to be $42 \%$. The catalytic converter wire mesh shrinks $42 \%$ higher HC emission than the original catalytic converter.

The effect of chemical and with the higher wire mesh 


\section{Emission Control In Diesel Engine Using Magnesium As A Catalyst}

substrate area are the major cause of it. The magnesium catalyst materials are $75 \%$ cheaper than noble metals. The exhaust emissions for the catalytic converters at engine operating condition with a constant $50 \%$ throttle and $3000 \mathrm{rpm}$. It is found that $\mathrm{HC}$ reduces from to extent of $52 \%$ with the help of Wire mesh Catco. Similar reductions of NOx and $\mathrm{CO}$ emissions are found in Wire mesh $\mathrm{Catco}(\mathrm{s})$. The wire mesh substrate's surface area was found to be about 25 times higher than the ceramic substrate.

\section{CONCLUSION}

By alluding through numerous papers and direction, the effectiveness of reactant decrease can be relied upon to increment by magnesium. The impetus expands at the scale of response by reactant's adsorption rate in such a structure, that the enactment vitality for response is diminished far beneath its incentive in un-catalyzed catalysed response. Magnesium metal is chosen for the present work as it is less expensive than rhodium, palladium and platinum. Additionally it adsorbs the reactants atom firmly enough to hold and dynamic the reactants yet not all that emphatically that the item can't breakaway likewise the dissemination of reactants and items into and out of the pore structure of magnesium occurred productively. Because of this, the contamination level for the fumes outflow of S.I. The motor has observed to be diminished which is better with the nano-sized exhaust system. Exhaust systems dependent on a splash of magnesium nano-molecule on copper strainer show unrivalled execution. Nano-molecule shows high-temperature steadiness past that regular experience in exhaust system applications. The above work additionally opens a pathway for some future prospects, for example, fumes gas distribution demonstrate for a decrease of NOx fixation level which is as of now accessible and must be joined with present exhaust system display and tried against investigations. Exhaust system dependent on a shower of magnesium Nano-molecule utilizing splash pyrolysis process. Nano-molecule shows high-temperature strength past that regular experience in exhaust system applications..

The accompanying ends drawn from the present investigation are given below.

- Developed a wire mesh based substrate with Magnesium based catalytic converter has been successful.

- The surface area of the wire mesh substrate is approximately 25 times greater than the ceramic substrate.

- The NOx conversion efficiency catalytic converters for wire mesh was found to be $96 \%$ when related with the existing exhaust system.

- The wire mesh catalytic converters had CO conversion efficiency around $75 \%$ and it shrinks $41 \%$ higher than the existing exhaust system.
[3] Forzatti P, Lietti L 1999 -Catalyst deactivation, Catalysis Today 52: $165-181$.

[4] Heywood J B 1989 - Internal combustion engine fundamentals, (New York: McGraw-Hill).

[5] KALAM, H MASJUKI, M REDZUAN, -Development and test of a new catalytic converter for natural gas fuelled engine. $\mathrm{S}^{-}$adhan $^{-}$a Vol. 34, Part 3, June 2009, pp. 467- 481.

[6] Kaspar J, Fornasiero P, Graziani M - Use of CeO2-based oxides in the three-way catalysis, Catalysis Today 50: 285-298.

[7] Kenji Yamauchi, Norihiro Murayama and Junji Shibata, -Absorption and Release of Carbon Dioxide with Various Metal Oxides and Hydroxides Materials Transactions, Vol. 48, No. 10 (2007) pp. 2739 to 2742.

[8] Me i-Rong Songa,b,c, Miao Chena, Zhi-Jun Zhangb , _Preparation and characterization of $\mathrm{Mg}$ nanoparticles. MATERIALS CHARACTERIZATION 59 (2008) $514-518$

[9] Mordike, Ebert ,Magnesium Properties - applications potentiall,Materials Science and Engineering A302 (2001) 37-45.

[10] Murali Krishna, Kishor , Murthy, Gupta and Narasimha Kumar,-Comparative Studies on Emissions from Two Stroke Copper Coated Spark Ignition Engine with Alcohols with Catalytic Converter, International Journal of Scientific \& Technology Research Volume 1, Issue 2, March 2012 ISSN 2277-8616

[11] Pranav Raghav Sood - Air Pollution Through Vehicular Emissions in Urban India and Preventive Measures IPCBEE vol.33 (2012) ( (2012) IACSIT Press, Singapore

[12] Taku Iwaoka and Mitsuru Nakamura, -Effect of Compaction Temperature on Sinterability of Magnesium and Aluminum Powder Mixtures by Warm 41 Compaction Method Materials Transactionsl, Vol. 52, No. 5 (2011) pp. 943 to 947

[13] Thakur Mukesh and Saikhedkar N.K.,"Reduction of Pollutant Emission from Two-wheeler Automobiles using Nano-particle as a Catalyst Res. J. Engineering Sci.Vol. 1(3), 32-37, Sept. (2012)

[14] Zissis Samaras, Karl-Heinz Zierock, - Emission Inventory Guidebook, Chapter 0706, Gasoline Evaporation from Vehicles\| Technical report No 16/2007, European Environment Agency, Copenhagen, Denmark, p. 21

\section{AUTHORS PROFILE}

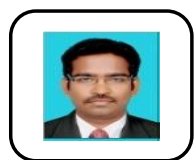

S.Yuvaraja, Assistant Professor, School of Mechanical Engineering, Sathyabama Institute of Science \& Technology, Chennai, India.

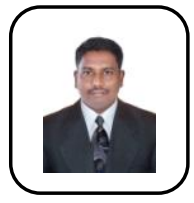

S.Venkatesh, Assistant Professor, School of Mechanical Engineering, Sathyabama Institute of Science \& Technology, Chennai, India..

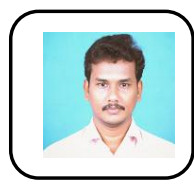

P.Karthikeyan, Assistant Professor, School of Mechanical Engineering, Sathyabama Institute of Science \& Technology, Chennai, India.

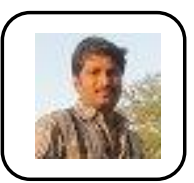

R.Kumarasubramanian, Assistant Professor, School of Mechanical Engineering, Sathyabama Institute of Science \& Technology, Chennai, India.

\section{REFERENCES}

[1] Angelidis T N, Papadakis P G 1997 - Partial regeneration of an aged commercial automotive catalyst -, Environmental 12: 193-206.

[2] Bonnel, Martini, Krasenbrink, -EURO 3 Stage for motorcycles: Derivation of equivalent limits for the WMTC driving cyclel, Joint Research Centre, Ispra, Italy, p.40 (Moto_103) 